Research Article

Ming-Cui Li and Ai-Xi Chen*

\title{
Enhanced Entanglement in Hybrid Cavity Mediated by a Two-way Coupled Quantum Dot
}

https://doi.org/10.1515/phys-2020-0003

Received Sep 20, 2019; accepted Jan 05, 2020

\begin{abstract}
We investigate theoretically the entanglement in a hybrid Fabry-Perot cavity system. A membrane in the cavity acts as a mechanical resonator, and a two-level quantum dot is coupled to both the cavity mode and the mechanical resonator. The entanglements between the cavity field and the mechanical resonator, between the mechanical resonator and the quantum dot, as well as between the cavity field and the quantum dot are observed. The logarithmic negativities in the first two subsystems are much larger than those in the system without two-way coupled quantum dot, and the entanglements are robust against the thermal temperature (entanglements still exist in tens of Kelvin). We also find that without direct coupling between the cavity field and the mechanical resonator, one can till observe effective entanglement between them in our system. Our work is helpful and may have potential applications in the research of multipartite entanglement in physical system.
\end{abstract}

Keywords: quantum entanglement; quantum dot; coherent manipulation

\section{Introduction}

Entanglement [1], a fundamental phenomenon of quantum mechanics, has been considered to be a crucial resource for quantum communication [2] and information processing [3]. Up to now, quantum entanglement has been prepared and manipulated in many physical systems, such as photons [4, 5], individual atoms [6], ions [7], between optical photon and solid-state spin qubit [8], etc. Optomechanical systems offer new types of control over the quantum states of both light and matter. By creating

\footnotetext{
*Corresponding Author: Ai-Xi Chen: School of Science, Zhejiang Sci-Tech University, Hangzhou 310018, China;

Email: aixichen@zstu.edu.cn

Ming-Cui Li: College of Information School, East China Jiaotong University, Nanchang 330013 China
}

entanglement between mechanical modes, hybrid optical cavity systems have good potential applications in quantum information processing. Vitali etc. [9] first show the stationary entanglement between an optical cavity field mode and a macroscopic vibrating mirror in a Fabry-Perot cavity. Many other types of entanglement are, afterwards, also studied and experimental demonstrated, such as entanglement between exciton and mechanical modes [10], between two vibrating mirrors [11], between tow remote micromechanical oscillators [12], etc. Hybrid optical cavities provide more controllable degrees of freedom; help us investigate various properties of the compound system, for instances, investigating the nonlinear properties of cavity optomechanics by introducing into the setup a quantum two-level system $[13,14]$, studying the atom-cavity entanglement with low excitation limit approximation of the atom ensembles [15], analyzing the entanglement between the optical cavity and the nanomechanical resonator beam via a quantum dot [16]. By including an optical parametric amplifier in an optical cavity, Yang et al. [40], Ahmed et al. [41] present schemes to enhance the optomechanical entanglement and the entanglement of two optical modes.

The typical schematic to study the interaction between optical cavities and mechanical objects is a Fabry-Perot cavity in which one of the cavity's mirrors is free to move. In fact, in Fabry-Perot cavity and other analogous cavities, the movable mirror must provide both optical confinement and mechanical pliability. Membrane-in-Cavity designs remove the need to integrate good mirrors into good mechanical devices [17, 18]. Meanwhile, varieties of devices integrating quantum dots and membranes are investigated and experimental demonstrated [19-23]. And many theoretical study and experiments of the coupling between twolevel system and mechanical resonator have proposed and demonstrated, such as strong coupling between a single trapped atom and a mechanical oscillator [24], coupling of an atomic force microscope cantilever to a quantum dot [25], nitrogen-vacancy defect coupled to a nanomechanical oscillator [26], exciton-phonon coupling with carbon nanotubes [27], coupling Josephson junction quantum circuits to micromechanical resonator [28], quantum dots in graphene nanoribbon and graphene sheet $[29,30]$,

Ә Open Access. (c) 2020 M.-C. Li and A.-X. Chen, published by De Gruyter. (cc) BY Attribution 4.0 License 
exciton-phonon coupling with a quantum dot embedded within a nanowire [31-33], spin-mechanical coupling of a quantum dots integrated into a cantilever resonator [34].

Motivated by these developments, we consider a hybrid Membrane-in-Cavity with a quantum dot coupling both with the optical cavity and the resonator. We study the entanglement of the system in three subsystems: cavity field and mechanical resonator subsystem, mechanical resonator and quantum dot subsystem, cavity field and quantum dot subsystem. We find that there are effective entanglements in these subsystems, and the entanglements in the first two subsystems are much strong compared with those in the system without the two-way coupled quantum dot. What's more, the entanglements in the first two subsystems are robust to temperature.

The rest of this paper is organized as follows. In Sec. 2, we introduce the model and describe the Hamiltonian of our system. In Sec. 3, the quantum Langevin equations are provided and the drift matrix, which is crucial in the Lyapunov equation to calculate the logarithmic negativity, is figured out. In Sec. 4, we calculated numerically the logarithmic negativities in the three subsystems and analyses in detail the dependence of the effective entanglement on thermal bath temperature, pump power, detuning, coupling strength,etc. Conclusions are given in Sec. 5.

\section{Model}

The model considered here is schematically depicted in Figure 1. The membrane is suspended within a high- $Q$ Fabry-Perot cavity which is driven by an intense pump laser of frequency $\omega_{l}$. Both mirrors of the cavity are fixed, and the membrane with a quantum dot serves as a membrane resonator in the cavity. We consider a single cavity mode of frequency $\omega_{c}$. This cavity mode is coupled to the

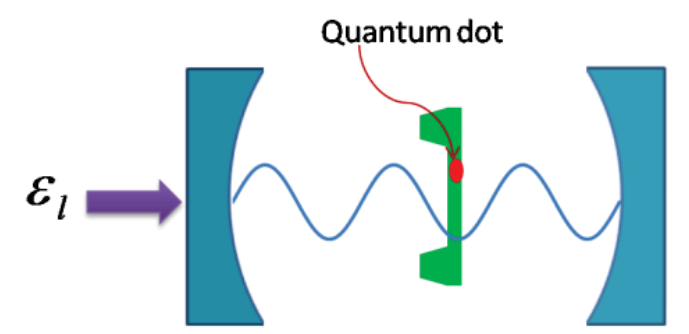

Figure 1: Schematic diagram of the optomechanical system. The two mirrors (blue) of the cavity are fixed. The membrane (green) inside the cavity oscillates at. The quantum dot (red) is coupled with both the cavity and the membrane. The cavity is also driven by a laser of frequency. membrane resonator mode (MR) via radiation pressure depending on both the photon number of the cavity and the displacement of the MR. The two-level quantum dot interacts with both the cavity and the MR.

The total Hamiltonian of the system is given by

$$
\begin{aligned}
H & =\hbar \omega_{c} c^{\dagger} c+\frac{1}{2} \hbar \omega_{m}\left(p^{2}+q^{2}\right)+\frac{1}{2} \hbar \omega_{e} s^{z} \\
& -\hbar g_{o m} c^{\dagger} c q+\frac{1}{2} \hbar g_{e m} s^{z} q+\hbar \mathrm{g}_{o e}\left(c^{\dagger}+c\right) \mathrm{s}_{x} \\
& +i \hbar \varepsilon_{l}\left(c^{\dagger} e^{-i \omega_{l} t}-c e^{i \omega_{l} t}\right)
\end{aligned}
$$

The first term represents the free energy of the cavity mode where $c^{\dagger}(c)$ is the creation (annihilation) operator satisfying the commutation relation $\left[c, c^{\dagger}\right]=1$. The second term is the energy of MR, described by the dimensionless position and momentum operators and $q$ which satisfy the commutation relation $[q, p]=i$. $p$ is defined by $p=\sqrt{\frac{1}{m \hbar \omega_{m}}} p_{o}$ and $q=\sqrt{\frac{m \omega_{m}}{\hbar}} q_{o}$ where $p_{o}\left(q_{o}\right)$ is the orig inal position (momentum) operator. The Hamiltonian of the localized quantum dot in MR is described by $\hbar w_{e} S^{z}$ with the transition frequency $\omega_{e} . s^{z}, s_{x}, s^{+}$and $s$ are operators used to describe the two-level quantum dot satisfying relations $\left[s^{z}, s^{+}\right]=2 s^{+},\left[s^{z}, s\right]=-2 s,\left[s^{+}, s\right]=s^{z}$ and $s_{X}=s^{+}+s$. The fourth term describes the coupling of the cavity mode with the MR. The interaction between the resonator and quantum dot is given by the fifth term in Eqs. (1), and $g_{e m}$ is the coupling strength [27]. The sixth term describes the coupling between the two-level system and the cavity with the coupling strength $g_{\text {oe }}[27,28]$. The last term describes the interaction of the cavity mode with the pump laser. The amplitude of the pump laser is defined by $\varepsilon_{l}=\sqrt{2 \kappa P_{l} /\left(\hbar w_{l}\right)}$, where $\kappa$ being the cavity decay rate and $P_{l}$ the input power.

In the frame rotating at the driving laser frequency $\omega_{l}$, applying a unitary transformation $U=\exp \left[-\frac{i}{\hbar}\left(\hbar \omega_{l} c^{\dagger} c+\right.\right.$ $\left.\left.\frac{1}{2} \hbar \omega_{l} s^{z}\right) t\right]=\exp \left(-i \omega_{l} c^{\dagger} c t-i \frac{1}{2} \omega_{l} s^{z} t\right)$ and applying the rotating wave approximation, one can obtain the Hamiltonian of the system as follows,

$$
\begin{aligned}
H & =\hbar \Delta_{c} c^{\dagger} c+\frac{1}{2} \hbar \omega_{m}\left(p^{2}+q^{2}\right)+\frac{1}{2} \hbar \Delta_{e} s^{z} \\
& -\hbar g_{o m} c^{\dagger} c q+\frac{1}{2} \hbar g_{e m} s^{z} q+h g_{o e}\left(c^{\dagger} s+c s^{\dagger}\right)+i \hbar \varepsilon_{l}\left(c^{\dagger}-c\right),
\end{aligned}
$$

where $\Delta_{c}=\omega_{c}-\omega_{l}$ and $\Delta_{e}=\omega_{e}-\omega_{l}$ are the detuning of the cavity mode and quantum dot with respect to the pump field.

\section{Calculation}

There are three main components optical cavity, mechanical resonator and quantum dot in our model. In this sec- 
tion, we calculate the entanglement between each two components in the system. Here, we consider the entanglements under the condition of low excitation limit approximation of the quantum dot which is broadly used in the analysis of the behavior of a hybrid cavity [13-15].

In the low quantum dot excitation limit, one has $s_{z} \simeq$ $\left\langle s_{z}\right\rangle \simeq-1$. And operator $s$ and $s^{z}$ satisfy the usual bosonic commutation relation $\left[s, s^{+}\right]=1$. In this case, the quantum Langevin equations describing the dynamic of the system then can be written as,

$$
\begin{gathered}
\dot{q}=\omega_{m} p, \\
\dot{p}=-\gamma_{m} p-\omega_{m} q+g_{o m} c^{\dagger} c+\frac{1}{2} g_{e m}+\xi, \\
\dot{c}=-\left[\kappa+i\left(\Delta_{c}-g_{o m} q\right)\right] c-i g_{o e} s+\varepsilon_{l}+\sqrt{2 \kappa} c_{i n}, \\
\dot{s}=-\left[\gamma_{a}+i\left(\Delta_{e}+g_{e m} q\right)\right] s-i g_{o e} c+\sqrt{2 \gamma_{a}} s_{i n} .
\end{gathered}
$$

Here $\gamma_{a}$ is the decay rate of quantum dot. $\xi(t)$ is the quantum Brownian noise acting on the MR. $c_{\text {in }}(t)$ and $s_{i n}(t)$ are input vacuum noise operators for the cavity and the quantum dot. They all have zero mean values $\langle\xi(t)\rangle=$ $\left\langle c_{i n}(t)\right\rangle=\left\langle s_{i n}(t)\right\rangle=0$. The non-zero correlation functions in time domain can be written as $[35,36]$

$$
\begin{gathered}
\left\langle\xi(t) \xi\left(t^{\prime}\right)\right\rangle=\frac{1}{2 \pi} \frac{\gamma_{m}}{\omega_{m}} \int \omega e^{-\omega\left(t-t^{\prime}\right)}[1 \\
\left.+\operatorname{coth}\left(\frac{h \omega}{2 k_{B} T}\right)\right] d \omega \\
\left\langle c_{\text {in }}(t){c_{\text {in }}}^{+}\left(t^{\prime}\right)\right\rangle=\delta\left(t-t^{\prime}\right) \\
\left\langle s_{\text {in }}(t){s_{\text {in }}}^{+}\left(t^{\prime}\right)\right\rangle=\delta\left(t-t^{\prime}\right),
\end{gathered}
$$

where $k_{B}$ is the Boltzmann constant and $T$ the environment temperature of MR. Under the condition of large mechanical quality factor limit of $Q=\omega_{m} / \gamma_{m} \gg 1, \xi(t)$ becomes delta correlated,

$$
\left\langle\xi(t) \xi\left(t^{\prime}\right)+\xi\left(t^{\prime}\right) \xi(t)\right\rangle / 2 \simeq \gamma_{m}\left(2 n_{t h}+1\right) \delta\left(t-t^{\prime}\right)
$$

where $n_{t h}=\left[\exp \left(\hbar \omega_{m} / k_{B} T\right)-1\right]^{-1}$ is the mean thermal excitation number.

We focus on the steady-state entanglement between different parts of our system, which can be analyzed from the dynamics of the fluctuations of the operators in the system. We first get the steady-state solutions of the operators by setting the time derivatives to zero in Eqs. (3)-(6) and calculate the mean values of the operators from the algebraic equations,

$$
\begin{gathered}
p_{s}=0, \\
q_{s}=\frac{g_{o m} I_{c}+\frac{1}{2} g_{e m}}{\omega_{m}}, \\
s_{s}=\frac{-i g_{o e}}{\gamma_{a}+i\left(\Delta_{e}+g_{e m} q_{s}\right)} c_{s},
\end{gathered}
$$

$c_{S}$

$$
=\frac{\varepsilon_{l}}{k+\frac{g_{o e} \gamma_{a}}{\gamma_{a}{ }^{2}+\left(\Delta_{e}+g_{e m} q_{s}\right)^{2}}+i\left[\Delta_{c}-g_{o m} q_{s}-\frac{g_{o e^{2}}\left(\Delta_{e}+g_{e m} q_{s}\right)}{\gamma_{a}+\left(\Delta_{e}+g_{e m} q_{s}\right)^{2}}\right]}
$$

where $I_{C}=\left|c_{S}\right|^{2}$ is the mean intracavity photon number in the steady state.

The dynamics of the fluctuation of the operators in the system is obtained from Eqs. (3)-(6) by decomposing each operator as the sum of its steady-state value and a small fluctuation, that is, $q=q_{s}+\delta q, p=p_{s}+\delta p$, $c=c_{s}+\delta c, s=s_{s}+\delta s$ and $s^{z}=s_{s}^{z}+\delta s^{z}$. Neglecting the higher-order fluctuations, we get the linearized quantum Langevin equations,

$$
\delta \dot{q}=\omega_{m} \delta p,
$$

$$
\begin{aligned}
\delta \dot{p} & =-\gamma_{m} \delta p-\omega_{m} \delta q+g_{o m} c_{s}{ }^{\star} \delta c+g_{o m} c_{s} \delta c^{\dagger}+\xi, \\
\delta \dot{c} & =-\left[\kappa+i\left(\Delta_{c}-g_{o m} q_{s}\right)\right] \delta c+i g_{o m} c_{s} \delta q-i g_{o e} \delta s \\
& +\sqrt{2 \kappa} c_{i n}
\end{aligned}
$$

$$
\begin{aligned}
\delta \dot{s} & =-\left[\gamma_{a}+i\left(\Delta_{e}+g_{e m} q_{s}\right)\right] \delta s-i g_{e m} s_{s} \delta q-i g_{o e} \delta c \\
& +\sqrt{2 \gamma_{a}} s_{i n}
\end{aligned}
$$

We are interested in the entanglements in terms of quadrature operators which are defined by $\delta X_{c}=\left(\delta c^{\dagger}+\right.$ $\delta c) / \sqrt{2}, \delta Y_{c}=i\left(\delta c^{\dagger}-\delta c\right) / \sqrt{2}, \delta X_{a}=\left(\delta s^{\dagger}+\delta s\right) / \sqrt{2}$ and $\delta Y_{a}=i\left(\delta s^{\dagger}-\delta s\right) / \sqrt{2}$. The noises are defined in the similar way $X_{c i n}=\left(c_{i n}^{\dagger}+c_{i n}\right) / \sqrt{2}, Y_{c i n}=i\left(c_{i n}^{\dagger}-c_{i n}\right) / \sqrt{2}$, $X_{\text {ain }}=\left(s_{i n}^{\dagger}+s_{i n}\right) / \sqrt{2}$ and $Y_{\text {ain }}=i\left(s_{i n}^{\dagger}-s_{i n}\right) / \sqrt{2}$. Eqs. (15)(18) can be rewritten in terms of $\delta q, \delta p, \delta X_{c}, \delta Y_{c}, \delta X_{a}$ and $\delta Y_{a}$ as follows.

$$
\delta \dot{q}=\omega_{m} \delta p
$$

$$
\delta \dot{p}=-\omega_{m} \delta q-\gamma_{m} \delta p+\frac{g_{o m}\left(c_{s}^{\star}+c_{S}\right)}{\sqrt{2}} \delta X_{c}
$$




$$
\begin{gathered}
+i \frac{g_{o m}\left(c_{s}^{\star}-c_{s}\right)}{\sqrt{2}} \delta Y_{c}+\xi . \\
\delta \dot{X}_{c}=\frac{i g_{o m}\left(c_{s}-c_{s}^{\star}\right)}{\sqrt{2}} \delta q-\kappa \delta X_{c}+\left(\Delta_{c}-g_{o m} q_{s}\right) \delta Y_{c} \\
+g_{o e} \delta Y_{a}+\sqrt{2 \kappa} X_{c i n} . \\
\delta \dot{Y}_{c}=\frac{g_{o m}\left(c_{s}^{\star}+c_{s}\right)}{\sqrt{2}} \delta q-\left(\Delta_{c}-g_{o m} q_{s}\right) \delta X_{c}-\kappa \delta Y_{c} \\
-g_{o e} \delta X_{a}+\sqrt{2 \kappa} Y_{c i n} . \\
\delta \dot{X}_{a}=\frac{i g_{e m}\left(s_{s}^{\star}-s_{s}\right)}{\sqrt{2}} \delta q+g_{o e} \delta Y_{c}-\gamma_{a} \delta X_{a} \\
+\left(\Delta_{e}+g_{e m} q_{s}\right) \delta Y_{a}+\sqrt{2 \gamma_{a}} X_{a i n} . \\
\delta \dot{Y}_{a}=-\frac{g_{e m}\left(s_{s}^{\star}+s_{s}\right)}{\sqrt{2}} \delta q-g_{o e} \delta X_{c}-\left(\Delta_{e}+g_{e m} q_{s}\right) \delta X_{a} \\
-\gamma_{a} \delta X_{a}+\sqrt{2 \gamma_{a}} Y_{a i n} .
\end{gathered}
$$

By using the vector of quadrature fluctuations $U^{T}=\left(\delta q, \delta p, \delta X_{c}, \delta Y_{c}, \delta X_{a}, \delta Y_{a}\right)$ and the noise vector $N^{T}=\left(0, \xi, \sqrt{2 \kappa} X_{c i n}, \sqrt{2 \kappa} Y_{c i n}, \sqrt{2 \gamma_{a}} X_{a i n}, \sqrt{2 \gamma_{a}} Y_{\text {ain }}\right)$, we can write Eqs. (19)-(24) as

$$
\dot{U(t)}=A U(t)+N(t)
$$

where $A$ is the drift matrix, and can be expressed as

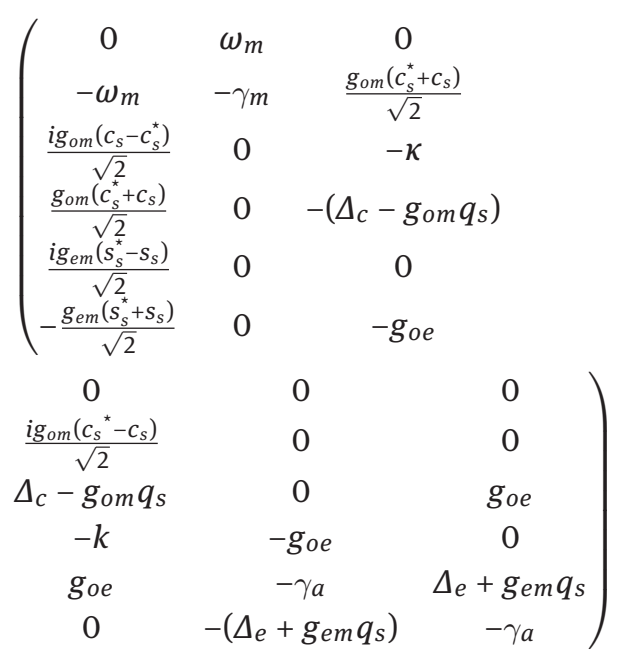

The stability conditions of the system can be derived by applying the Routh-Huiwitz criteria [37]. However, it is too cumbersome to list out here. We instead guarantee the stability by numerically calculating the eigenvalues of the drift matrix $A$. When the real parts of all the eigenvalues of matrix $A$ are negative, the system is stable. The quantum steady state for the fluctuations is fully characterized by the correlation matrix $V_{i j}=$ $\left(\left\langle U_{i}(\infty) U_{j}(\infty)+U_{j}(\infty) U_{i}(\infty)\right\rangle\right) / 2$. When the system is stable, the correlation matrix satisfies Lyapunov equation $A V+V A^{T}=-D$. The elements of matrix $D$ are obtained using the correlations of the noise operators [9]. Here, $D$. The Lyapunov equation is a linear equation for the covariance matrix $V$ and can be solved straightforwardly. Here, we can write the covariance matrix $V$ as

$$
V=\left[\begin{array}{ccc}
V_{m} & V_{c m} & V_{m d} \\
V_{c m}^{T} & V_{c} & V_{c d} \\
V_{m d}^{T} & V_{c d}^{T} & V_{d}
\end{array}\right]
$$

Each element of matrix $V$ in Eq. (27) is a matrix, and the elements $V_{m}$, and $V_{d}$ on the main diagonal represent the variance of the three components in our system, i.e., mechanical resonator, cavity field and quantum dot. And the elements off diagonal indicate the covariance between each two components. To study the entanglement properties of the steady states of the cavity and the mechanical resonator, we extract a reduced correlation matrix $V_{r}$ from $V$, i.e., $V_{r}=\left(\begin{array}{cc}V_{m} & V_{c m} \\ V_{c m}^{T} & V_{c}\end{array}\right)$. We adopt logarithmic negativity $[38,39]$ which is widely used in numerical modeling because it is easy to calculate, measure the entanglement for mixed states, and multipartite states. For continuous variables, logarithmic negativity is defined as

$$
E N=\max [0,-\ln 2 \varsigma],
$$

where $\varsigma=2^{-1 / 2}\left[\sigma-\sqrt{\sigma^{2}-4 \operatorname{det} V_{r}}\right]^{1 / 2}$, with $\sigma=\operatorname{det} V_{c}+$ $\operatorname{det} V_{m}-2 \operatorname{det} V_{c m}$. The optical cavity and the mechanical resonator modes are entangled when the logarithmic negativity $E N$ is positive. Namely, a Gaussian state is entangled if and only if $\varsigma<1 / 2$, and that is consistent with Simon's theory [42]. The reduced correlation matrices for MR and quantum dot, cavity field and quantum dot are extracted, and their logarithmic negativities are calculated, in the same way.

\section{Entanglement between different components of the system}

We solve the Lyapunov equation and plot the logarithmic negativity of cavity field-MR subsystem $E N_{c m}$, MRquantum dot subsystem $E N_{m d}$ and cavity field-quantum dot subsystem $E N_{c d}$ as a function of temperature, of the input laser power, of the cavity field-pump laser detuning, of dot-pump laser detuning, of the cavity field-MR coupling strength, and of the coupling strength between MR and quantum dot, which are shown in Figure 2-7. The parameters are chosen with reference to the recent experiments. 

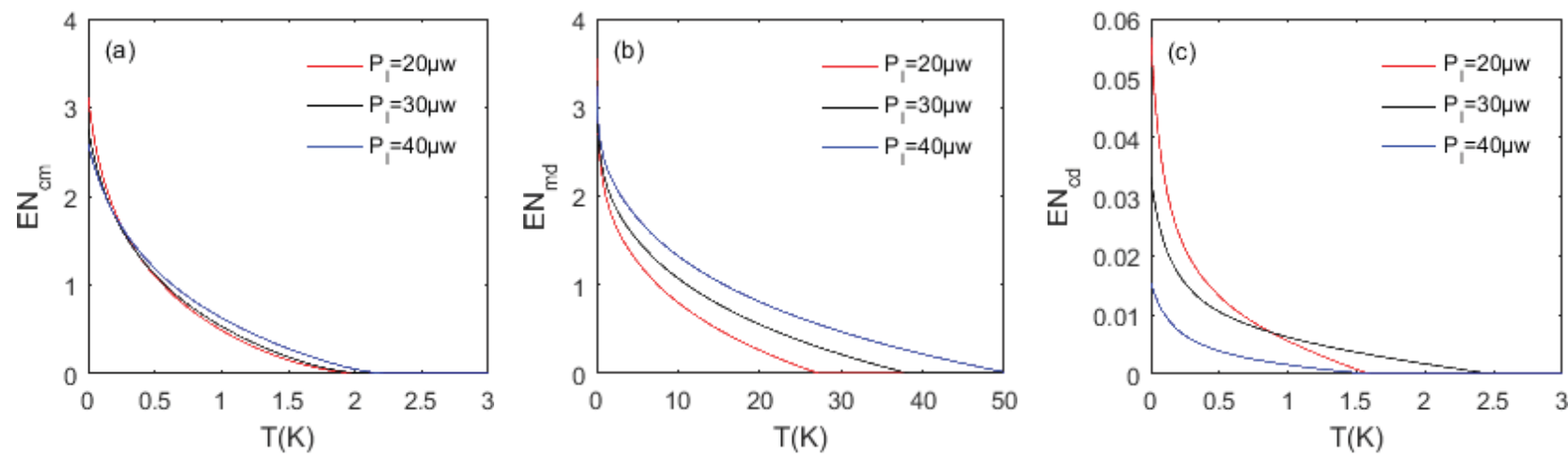

Figure 2: (a) logarithmic negativity $E N_{c m}$ of the cavity field-mechanical resonator subsystem; (b) $E N_{m d}$ of the mechanical resonatorquantum dot subsystem; (c) $E N_{c d}$ of the cavity field-quantum dot subsystem as a function of the temperature for input laser power $P_{l}=20 \mu \mathrm{w}, P_{l}=30 \mu \mathrm{w}$, and $P_{l}=40 \mu \mathrm{w}$ respectively. Other parameters are $\omega_{m} / 2 \pi=60 \mathrm{MHz}, \kappa=0.1 \omega_{m}, g_{o m}=3000 \mathrm{~Hz}, \gamma_{m}=227 \mathrm{~Hz}$, $g_{e m}=0.01 \omega_{m}, g_{o e}=0.06 \omega_{m}, \gamma_{a}=10 \mathrm{MHz}, \Delta_{e}=0.03 \omega_{m}$ and $\Delta_{c}=-0.1 \omega_{m}$.
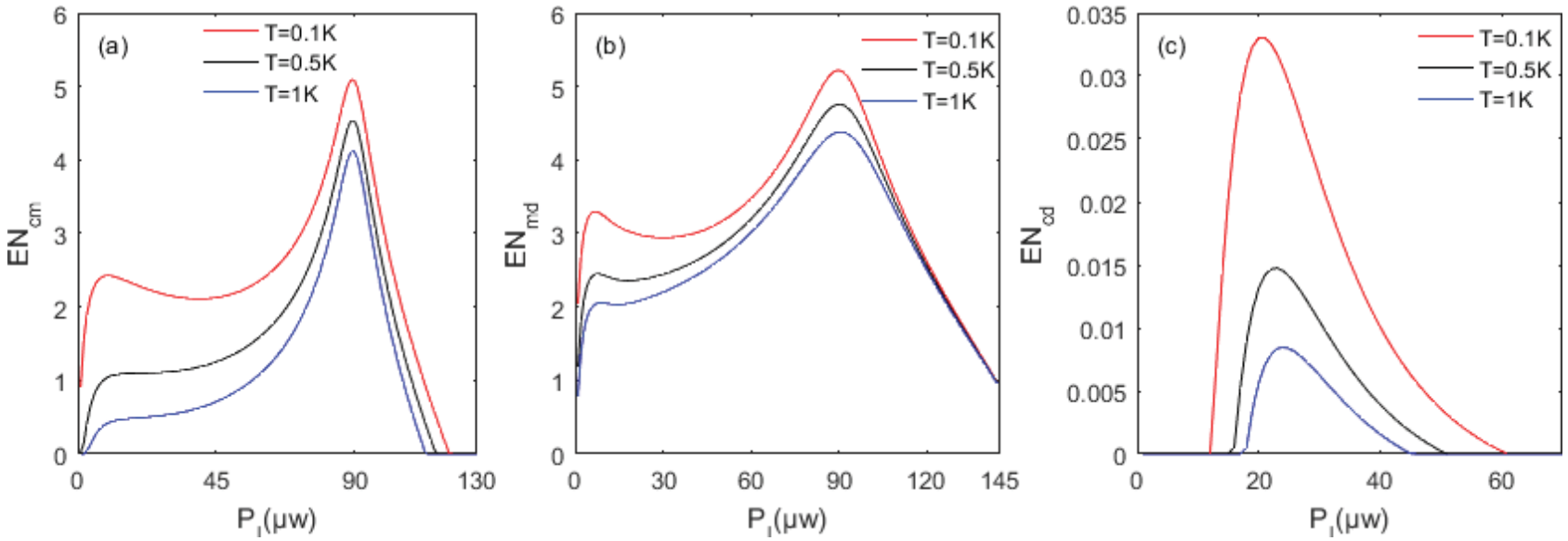

Figure 3: (a) logarithmic negativity $E N_{c m}$ of the cavity field-mechanical resonator subsystem; (b) $E N_{m d}$ of the mechanical resonatorquantum dot subsystem; (c) $E N_{c d}$ of the cavity field-quantum dot subsystem as a function of the input laser power for thermal bath temperature $T=0.1 \mathrm{~K}, T=0.5 \mathrm{~K}$, and $T=1 \mathrm{~K}$ respectively. Other parameters are the same as in Figure 2

The frequency of MR is $\omega_{m} / 2 \pi=60 \mathrm{MHz}$, and the quality of MR $Q \approx 10^{6}$.

In Figure 2, the cavity is weakly driven by a pump laser of input power of $20 \mu \mathrm{w}, 30 \mu \mathrm{w}$ and respectively, and the cavity field-MR coupling strength is $g_{\text {om }}=3000$, quantum dot-MR coupling strength is $g_{e m}=0.01 \omega_{m}$. We find that the entanglement of cavity field-MR subsystem and MR-quantum dot subsystem are much stronger than that of cavity field-quantum dot subsystem. This can be explained from the elements in the drift Matrix $A$. The effective interaction strength in the cavity field-MR subsystem is altered from $g_{\text {om }}$ to $\frac{g_{\text {om }}\left(c_{s}^{*}+c_{s}\right)}{\sqrt{2}}$ and $\frac{i g_{o m}\left(c_{s}^{*}-c_{s}\right)}{\sqrt{2}}$, and the effective coupling strength in the MR-quantum dot subsystem is also changed into and $-\frac{g_{e m}\left(s_{s}^{*}+s_{s}\right)}{\sqrt{2}}$. Nevertheless, the effective coupling strength in cavity field-quantum dot subsystem remain the same as $g_{o e}$. Thus, the effective coupling strength is enhanced in the first two subsystems, while the third one not. And an enhanced coupling strength is in favor of strong entanglement in most cases. One can also see from Figure 2 that the entanglement of MR-quantum dot subsystem is more robust against thermal bath temperature than those of the other two subsystems. These entanglements are, as expected, decrease with the increasing thermal bath temperature. The critical temperature, at which the entanglement disappears, increases as the increasing of input laser power as shown in Figure 2(a) and 2(b). However, it is not the case in Figure 2(c), which means there may be an optimal input laser power for the fixed detuning.

In Figure 3, we plot the logarithmic negativity of the three subsystems as a function of the input laser power for the fixed cavity field-pumper laser detuning $\Delta_{c}=-0.1 \omega_{m}$ and quantum dot-pump laser detuning $\Delta_{e}=0.03 \omega_{m}$. For the same input laser power, the entanglement decreases with the increasing of the temperature which is entirely 

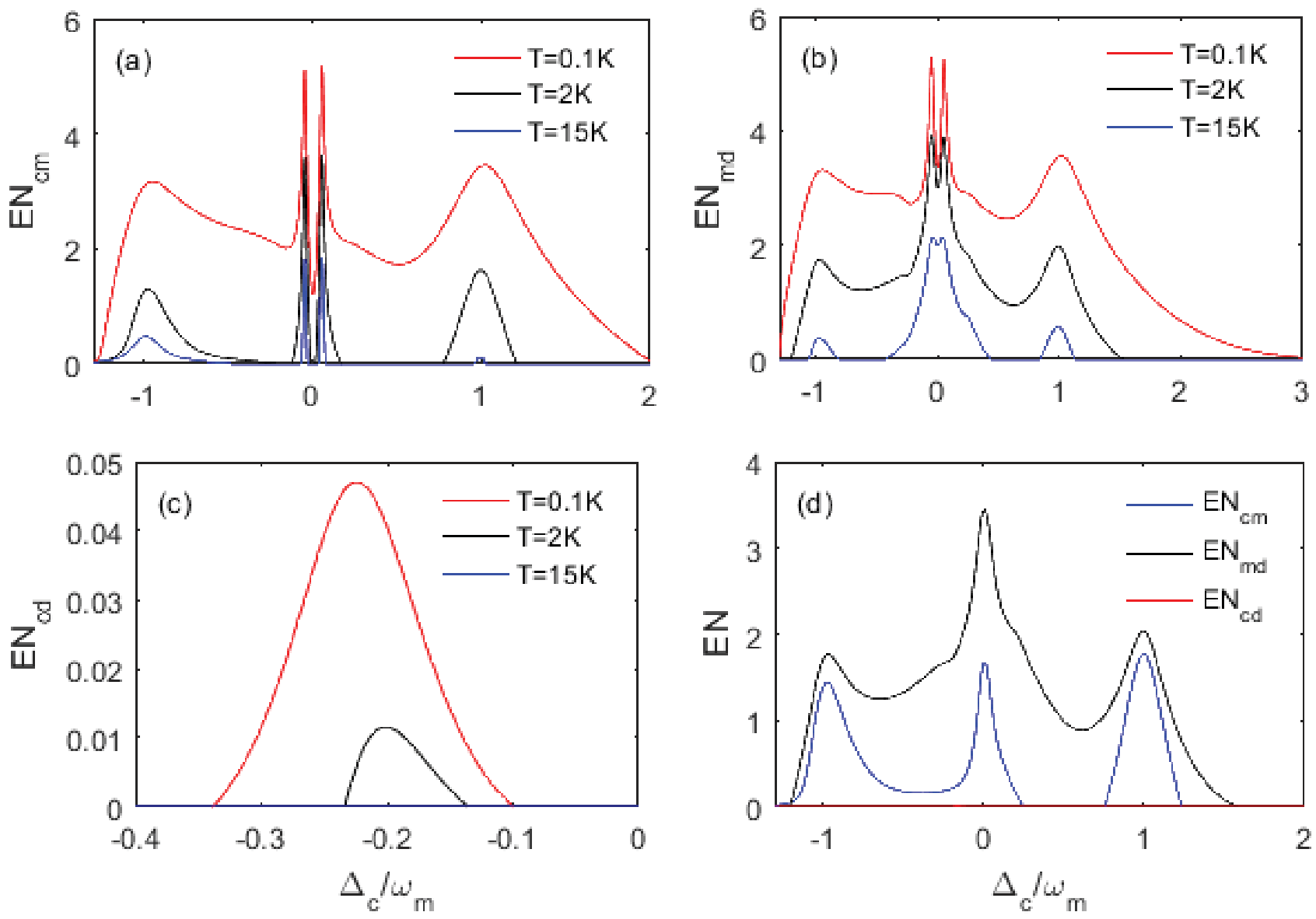

Figure 4: (a) Logarithmic negativity of the cavity field-mechanical resonator subsystem $E N_{c m}$; (b) of the mechanical resonator-quantum dot subsystem $E N_{m d}$; (c) of the cavity field-quantum dot subsystem $E N_{c d}$ as a function of the scaled cavity-pump laser detuning $\Delta_{c} / \omega_{m}$ for fixed quantum dot-pump laser detuning $\Delta_{e} / \omega_{m}$, fixed pump laser power $P_{l}=50 \mu w$ and different temperatures $T=0.1 \mathrm{~K}, T=2 \mathrm{~K}$, and $T=15 \mathrm{~K}$. (d) Logarithmic negativity of three subsystems for input power $P_{l}=30 \mu \mathrm{w}$ and $T=1 \mathrm{~K}$. Other parameters are the same as in Figure 2.
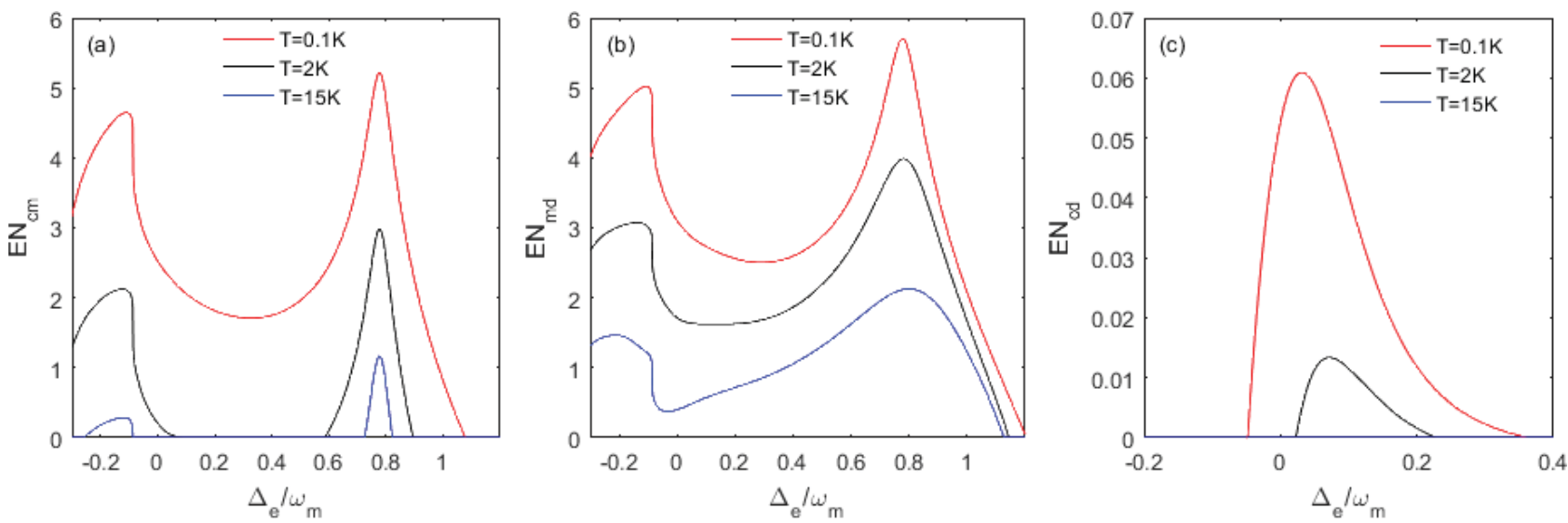

Figure 5: (a) logarithmic negativity of the cavity field-mechanical resonator subsystem $E N_{c m}$; (b) of the mechanical resonator-quantum dot subsystem $E N_{m d}$; (c) of the cavity field-quantum dot subsystem $E N_{c d}$ as a function of the scaled quantum dot-pump laser detuning $\Delta_{e} / \omega_{m}$ for fixed cavity-pump laser detuning $\Delta_{c}=-0.2 \omega_{m}$ and fixed input power $P_{l}=50 \mu \mathrm{w}$. Other parameters are the same as in Figure 2 . 

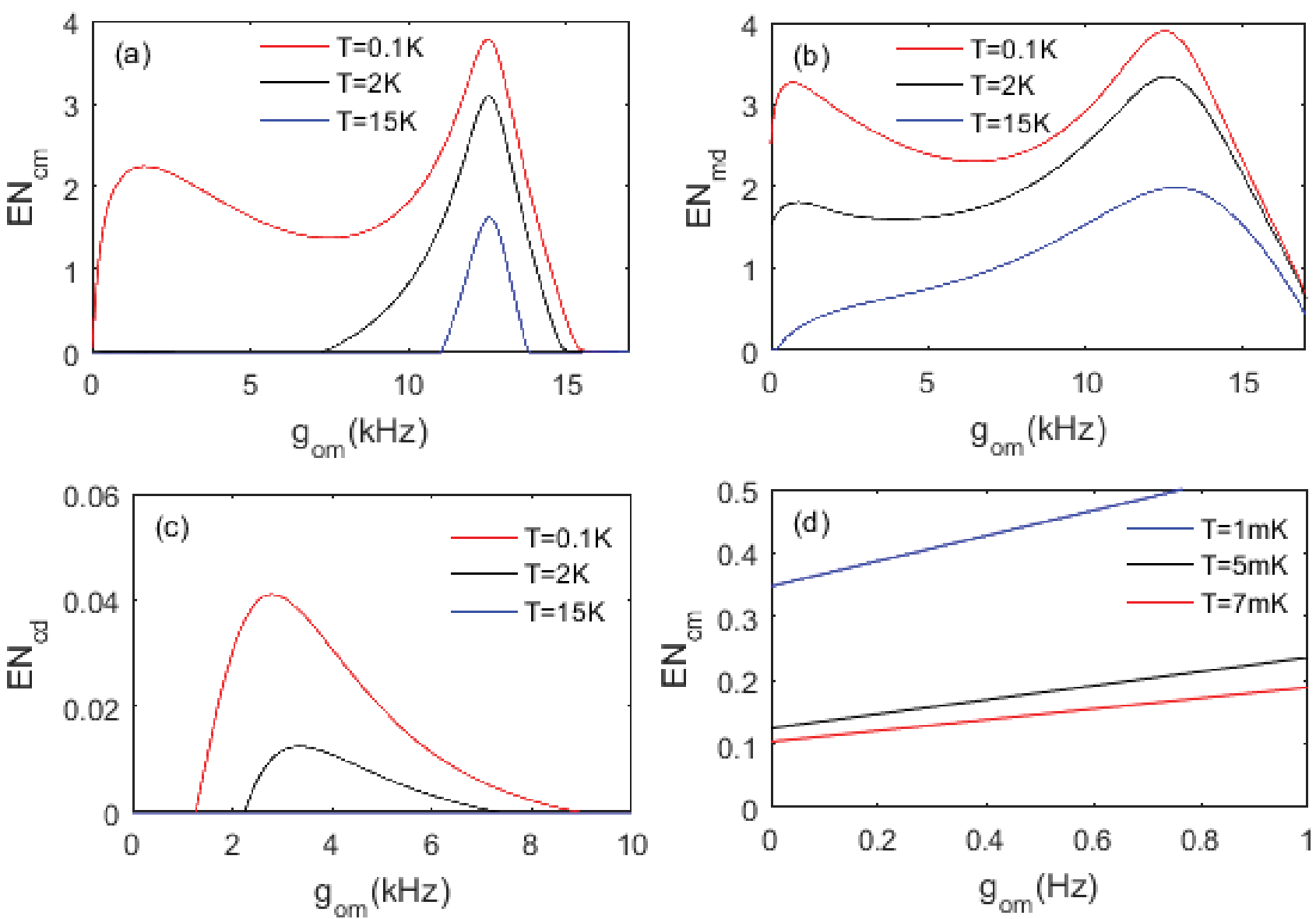

Figure 6: (a) Logarithmic negativity of the cavity field-mechanical resonator subsystem $E N_{c m}$; (b) of the mechanical resonator-quantum dot subsystem $E N_{m d}$; (c) of the cavity field-quantum dot subsystem $E N_{c d}$ as a function of the cavity mode-MR coupling strength $g_{o m}$ for different temperatures $T=0.1 \mathrm{~K}, T=2 \mathrm{~K}$, and $T=15 \mathrm{~K}$. (d) Logarithmic negativity of third subsystem for temperatures $T=1 \mathrm{mK}, T=5 \mathrm{mK}$ and $T=7 \mathrm{mK}$. The detuning are fixed at $\Delta_{e}=0.1 \omega_{m}, \Delta_{c}=-0.2 \omega_{m}$ and the pump laser power is fixed at $P_{l}=50 \mu w$. Other parameters are the same as in Figure 2.
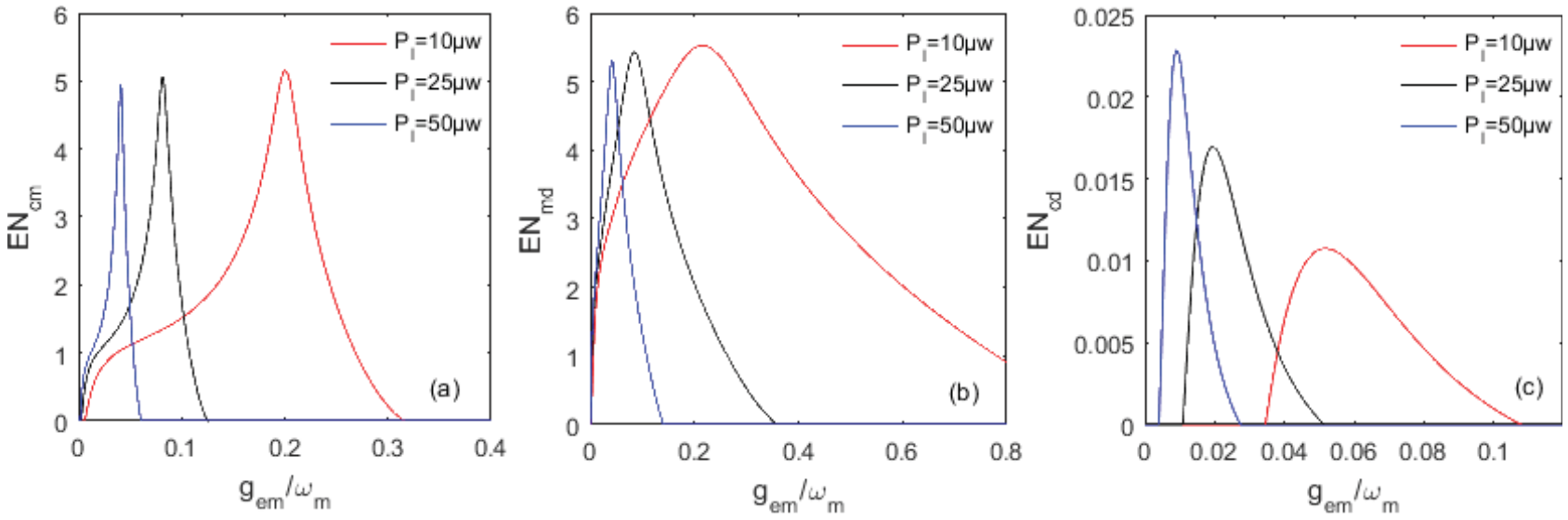

Figure 7: (a) logarithmic negativity of the cavity field-mechanical resonator subsystem $E N_{c m}$; (b) of the mechanical resonator-quantum dot subsystem $E N_{m d}$; (c) of the cavity field-quantum dot subsystem $E N_{c d}$ as a function of the scaled quantum dot-mechanical resonator coupling strength $g_{e m} / \omega_{m}$ for fixed detuning $\Delta_{c}=-0.2 \omega_{m}, \Delta_{e}=0.1 \omega_{m}$ and temperature $T=0.5 \mathrm{~K}$. Other parameters are the same as in Figure 2 . 
reasonable. Nevertheless, the entanglement does not increase linearly with the input power. There are two peaks in each curve in Figure 3(a) and 3(b). The first one locates at about $P_{l} \approx 10 \mu \mathrm{w}$ and the second one $P_{l} \approx 90 \mu \mathrm{w}$. And there is only one peak in each curve in Figure 3(c) located at $P_{l} \approx 25 \mu w$. That is, on the basis of the parameters in Figure 3, the optimal pump powers for cavity field-MR entanglement, MR-quantum dot entanglement and cavity fieldquantum dot entanglement are $90 \mu \mathrm{w}, 90 \mu \mathrm{w}$ and $25 \mu \mathrm{w}$ respectively. When the pump power exceeds $145 \mu \mathrm{w}$, the system becomes unstable. What's more, the logarithmic negativity in cavity field-MR, MR-quantum dot subsystem can reach 1 and 2 respectively for the input power $P_{l}=1 \mu \mathrm{w}$ at temperature $T=0.1 \mathrm{~K}$, while the entanglement in cavity filed-quantum dot subsystem does not appear until the input power increases to about $10 \mu \mathrm{w}$ and sharply decreased to zero $P_{l} \approx 60 \mu w$.

Figure 4 shows the entanglement of the three subsystems over the cavity field-pump laser detuning. We find that the main regime for effective entanglement in these three subsystems is within $\left[-1.3 \omega_{m}, 5 \omega_{m}\right]$, and the unstable regime lies mainly on the left side of the resonant point. The detuning regime for effective cavity fieldquantum dot entanglement is limited to a small regime of negative detuning near resonant. The detuning regimes for cavity field-MR and MR-quantum dot subsystem are much larger than that of cavity field-quantum dot subsystem. The peaks on the curves of these two subsystem are related to three particular detuning points $\Delta_{c}=-\omega_{m}, \Delta_{c}=0$, $\Delta_{c}=+\omega_{m}$. That is, when the pump laser is detuned to either the red sideband $\omega_{l}=\omega_{c}-\omega_{m}$ or the blue sideband $\omega_{l}=\omega_{c}+\omega_{m}$, one can get a local optimal entanglement of these subsystems. If the pump laser is resonant with the cavity, the entanglement increases gradually with the in put power. However, the peaks at $\Delta_{c}=0$ will split symmetrically when the input power exceeds $45 \mu w$. And the split is much sharper in cavity field-MR subsystem than that in MR-quantum dot subsystem as depicted in Figure 4(a) and $4(\mathrm{~b})$. If the input power increase to $70 \mu \mathrm{w}$, there will be a small unstable regime near the resonant point. This unstable regime expands slightly with the increasing of power, for instances the unstable regime for $P_{l}=70 \mu \mathrm{w}$ is $\left[-0.0294 \omega_{m}, 0.0475 \omega_{m}\right]$, and $\left[-0.0586 \omega_{m}, 0.0793 \omega_{m}\right]$ for $P_{l}=90 \mu \mathrm{w}$.

The influence of quantum dot-pump laser detuning $\Delta_{e}$ is demonstrated in Figure 5. Similar to that of $\Delta_{c}$, the stable detuning regimes of $\Delta_{e}$ for effective entanglement in cavity field-MR subsystem and MR-quantum dot subsystem are much wider than that in cavity field-quantum dot subsystem. And the optimal detuning region of the third subsystem is somewhat opposite to those of the former two sub- systems. That is, the entanglement in the third subsystem ascends gradually from $\Delta_{e} \approx 0.1 \omega_{m}$, and reach the maximum, and then declines to zero at $\Delta_{e} \approx 0.4 \omega_{m}$, while the direction of the curves in the first two subsystems is completely opposite in that detuning region.

In Figure 6, we plot the logarithmic negativities of the three subsystems as a function of the cavity field-MR coupling strength $g_{o m}$. We find that the entanglement in MRquantum dot subsystem is the most robust against $g_{o m}$ which is mainly due to the direct interaction between the mechanical resonator and quantum dot. There are two peaks on each curve of the first two subsystems at low temperatures in the stable regime for effective entanglement as shown in Figure 6(a) and 6(b). Nevertheless, the entanglement in cavity field-MR subsystem is much more sensitive to the change in temperature. When the thermal bath temperature increases to above $2 K$, there is only one peak remained on each curve in Figure 6 (a) with the location at $g_{\text {om }} \approx 12.5 \mathrm{kHz}$. Interestingly, we also find that the entanglement in cavity field-MR subsystem still exists when there is no direct coupling between the cavity field and MR, i.e., $g_{o m}=0$ under the condition of low temperature $T<1 K$. The logarithmic negativity $E N_{c m}$ is about 0.06 for $g_{o m}=0$ and $T=0.1 K$. In Figure 6(d), we can see that the logarithmic negativity in cavity field-MR subsystem is over o.1 if the temperature is under 7 milli-Kelvin with $g_{o m}=0$. And it increases to 0.35 when $T=1 \mathrm{mK}$. That means under the condition of low temperature, without direct coupling between the cavity field and mechanical resonator, the entanglements in cavity field-MR and MR-quantum dot subsystem are still robust. Thus, if we just consider the entanglement in the first two subsystems, the mechanical resonator in our system can have varieties of alternatives including those not so suitable for coupling by optical radiation pressure, such as graphene membrane, cantilever, carbon nano tube, etc.

The dependence of the entanglement of the three subsystems on the coupling strength between MR and quantum dot is depicted in Figure 7. In the stable regime, the optimal coupling strength for effective entanglement $E N_{c m}$, $E N_{m d}$ and $E N_{c d}$ are $g_{e m} \approx 0.04 \omega_{m}, g_{e m} \approx 0.05 \omega_{m}$ and $g_{\text {em }} \approx 0.01 \omega_{m}$ with the input power $P_{l}=50 \mu w$. The optimal coupling strength $g_{e m}$ decreases with the increase of the input power as shown in Figure 7, and the stable coupling regime also shrinks. Actually, each of the parameters in our system makes contribution to the effective entanglement in these three subsystems. All the parameters should be set a value in a stable regime. To get the desired entanglement in these subsystems, one should make a trade off among these parameters. 


\section{Conclusions}

In conclusion, we have studied in detail the stationary continuous-variable entanglement between the cavity field and the nanomembrane resonator, entanglement between the mechanical resonator and the two-level quantum dot, as well as entanglement between the cavity field and the quantum dot. The cavity with a membrane in it is weakly driven (with pump power less than $100 \mu w$ ) by a coherent light and mediated by a quantum dot. The quantum dot interacts both with the optical cavity and the membrane resonator.

We find that there are effective entanglements in all three subsystems. The entanglements in the first two subsystems are much stronger than that in the third subsystem mainly due to the enhanced interaction between the cavity field and the mechanical resonator, and the enhanced interaction between the mechanical resonator and the quantum dot. The logarithmic negativities in the first two subsystems can be up to about 7 which is much stronger than most of those in previous studies (often less than 1).

And the entanglements in the first two subsystems are robust to thermal bath temperature with effective entanglements exits at tens of Kelvin. We also find that the effective entanglement still exists when there is no direct opticalmechanical coupling at all in our system, which means one can obtain optical-mechanical entanglement through the help of coupling two-level system with mechanical resonator. What's more, the key to our system is a two-level system coupled to both the cavity field and the mechanical resonator, thus our model can be expanded to other physical systems which can accomplish this property. Our work is helpful and may have potential applications in the research of multipartite entanglement in physical systems.

Acknowledgement: This work is supported by the project of National Natural Science Foundation of China [grant number 11775190]; the project of Science and Technology of Jiangxi province [grant number 20171BAB212006]; the project of science and technology awarded by Education Bureau of Jiangxi Province [grant number JJ160503].

\section{References}

[1] Horodecki R, Horodecki P, Horodecki M, Horodecki K. Quantum entanglement. Rev Mod Phys. 2009;81(2):865-942.

[2] Kimble HJ. The quantum internet. Nature. 2008 Jun;453(7198): 1023-30.
[3] Nielsen MA, Chuang I. Quantum computation and quantum information. Cambridge, England: Cambridge University Press; 2002.

[4] Kwiat PG, Mattle K, Weinfurter H, Zeilinger A, Sergienko AV, Shih Y. New high-intensity source of polarization-entangled photon pairs. Phys Rev Lett. 1995 Dec;75(24):4337-41.

[5] Bowen WP, Treps N, Schnabel R, Lam PK. Experimental demonstration of continuous variable polarization entanglement. Phys Rev Lett. 2002 Dec;89(25):253601.

[6] Ritter S, Nölleke C, Hahn C, Reiserer A, Neuzner A, Uphoff M, et al. An elementary quantum network of single atoms in optical cavities. Nature. 2012 Apr;484(7393):195-200.

[7] Jost JD, Home JP, Amini JM, Hanneke D, Ozeri R, Langer C, et al. Entangled mechanical oscillators. Nature. 2009 Jun;459(7247):6835.

[8] Togan E, Chu Y, Trifonov AS, Jiang L, Maze J, Childress L, et al. Quantum entanglement between an optical photon and a solidstate spin qubit. Nature. 2010 Aug;466(7307):730-4.

[9] Vitali D, Gigan S, Ferreira A, Böhm HR, Tombesi P, Guerreiro A, et al. Optomechanical entanglement between a movable mirror and a cavity field. Phys Rev Lett. 2007 Jan;98(3):030405.

[10] Sete EA, Eleuch H, Ooi CH. Entanglement between exciton and mechanical modes via dissipation-induced coupling. Phys Rev A. 2015;92(3):033843.

[11] Ockeloen-Korppi CF, Damskägg E, Pirkkalainen JM, Asjad M, Clerk AA, Massel F, et al. Stabilized entanglement of massive mechanical oscillators. Nature. 2018 Apr;556(7702):478-82.

[12] Riedinger R, Wallucks A, Marinković I, Löschnauer C, Aspelmeyer $M$, Hong S, et al. Remote quantum entanglement between two micromechanical oscillators. Nature. 2018 Apr;556(7702):473-7.

[13] Sete EA, Eleuch H. Controllable nonlinear effects in an optomechanical resonator containing a quantum well. Phys Rev A. 2012;85(4):043824.

[14] Chang Y, Shi T, Liu YX, Sun CP, Nori F. Multistability of electromagnetically induced transparency in atom-assisted optomechanical cavities. Phys Rev A. 2011;83(6):063826.

[15] Genes C, Vitali D, Tombesi P. Emergence of atom-lightmirror entanglement inside an optical cavity. Phys Rev A. 2008;77(5):050307.

[16] Yuan XZ. Entangling an optical cavity and a nanomechanical resonator beam by means of a quantum dot. Phys Rev A. 2013;88(5):052317.

[17] Thompson JD, Zwickl BM, Jayich AM, Marquardt F, Girvin SM, Harris JG. Strong dispersive coupling of a high-finesse cavity to a micromechanical membrane. Nature. 2008 Mar;452(7183):72-5.

[18] Purdy TP, Peterson RW, Yu PL, Regal CA. Cavity optomechanics with Si3N4 membranes at cryogenic temperatures. New J Phys. 2012;14(11):115021.

[19] Gallo P, Felici M, Dwir B, Atlasov KA, Karlsson KF, Rudra $A$, et al. Integration of site-controlled pyramidal quantum dots and photonic crystal membrane cavities. Appl Phys Lett. 2008;92(26):263101.

[20] Zheng W, Liu Y, West A, Schuler EE, Yehl K, Dyer RB, et al. Quantum dots encapsulated within phospholipid membranes: phasedependent structure, photostability, and site-selective functionalization. J Am Chem Soc. 2014 Feb;136(5):1992-9.

[21] Niederbracht H, Hargart F, Schwartz M, Koroknay E, Kessler CA, Jetter $M$, et al. Fabrication and optical characterization of large scale membrane containing InP/AlGalnP quantum dots. Nanotechnology. 2015 Jun;26(23):235201. 
[22] Carter SG, Bracker AS, Yakes MK, Zalalutdinov MK, Kim M, Kim $\mathrm{CS}$, et al. Sensing flexural motion of a photonic crystal membrane with InGaAs quantum dots. Appl Phys Lett. 2017;111(18):183101.

[23] Capretti A, Lesage A, Gregorkiewicz T. Integrating Quantum Dots and Dielectric Mie Resonators: A Hierarchical Metamaterial Inheriting the Best of Both. ACS Photonics. 2017 Sep;4(9):2187-96.

[24] Hammerer K, Wallquist M, Genes C, Ludwig M, Marquardt F, Treutlein $\mathrm{P}$, et al. Strong coupling of a mechanical oscillator and a single atom. Phys Rev Lett. 2009 Aug;103(6):063005.

[25] Bennett SD, Cockins L, Miyahara Y, Grütter P, Clerk AA. Strong electromechanical coupling of an atomic force microscope cantilever to a quantum dot. Phys Rev Lett. 2010 Jan;104(1):017203.

[26] Arcizet O, Jacques V, Siria A, Poncharal P, Vincent P, Seidelin S. A single nitrogen-vacancy defect coupled to a nanomechanical oscillator. Nat Phys. 2011;7(11):879-83.

[27] Wilson-Rae I, Galland C, Zwerger W, Imamoğlu A. Excitonassisted optomechanics with suspended carbon nanotubes. New J Phys. 2012;14(11):115003.

[28] Pirkkalainen JM, Cho SU, Massel F, Tuorila J, Heikkilä TT, Hakonen PJ, et al. Cavity optomechanics mediated by a quantum two-level system. Nat Commun. 2015 Apr;6(1):6981.

[29] Wang S, Kharche N, Costa Girão E, Feng X, Müllen K, Meunier V, et al. Quantum dots in graphene nanoribbons. Nano Lett. 2017 Jul;17(7):4277-83.

[30] Qiao JB, Jiang H, Liu H, Yang H, Yang N, Qiao KY, et al. Bound states in nanoscale graphene quantum dots in a continuous graphene sheet. Phys Rev B. 2017;95(8):081409.

[31] Yeo I, de Assis PL, Gloppe A, Dupont-Ferrier E, Verlot P, Malik NS, et al. Strain-mediated coupling in a quantum dot-mechanical oscillator hybrid system. Nat Nanotechnol. 2014 Feb;9(2):10610.

[32] Montinaro M, Wüst G, Munsch M, Fontana $Y$, Russo-Averchi $\mathrm{E}$, Heiss $\mathrm{M}$, et al. Quantum dot opto-mechanics in a fully selfassembled nanowire. Nano Lett. 2014 Aug;14(8):4454-60.
[33] Munsch M, Kuhlmann AV, Cadeddu D, Gérard JM, Claudon J, Poggio $\mathrm{M}$, et al. Resonant driving of a single photon emitter embedded in a mechanical oscillator. Nat Commun. 2017 Jul;8(1):76.

[34] Carter SG, Bracker AS, Bryant GW, Kim M, Kim CS, Zalalutdinov MK, et al. Spin-mechanical coupling of an InAs quantum dot embedded in a mechanical resonator. Phys Rev Lett. 2018 Dec;121(24):246801.

[35] Wang G, Huang L, Lai YC, Grebogi C. Nonlinear dynamics and quantum entanglement in optomechanical systems. Phys Rev Lett. 2014 Mar;112(11):110406.

[36] Giovannetti V, Vitali D. Phase-noise measurement in a cavity with a movable mirror undergoing quantum Brownian motion. Phys Rev A. 2001;63(2):023812.

[37] Dejesus EX, Kaufman C. Routh-Hurwitz criterion in the examination of eigenvalues of a system of nonlinear ordinary differential equations. Phys Rev A Gen Phys. 1987 Jun;35(12):5288-90.

[38] Vidal G, Werner RF. Computable measure of entanglement. Phys Rev A. 2002;65(3):032314.

[39] Adesso G, Serafini A, Illuminati F. Extremal entanglement and mixedness in continuous variable systems. Phys Rev A. 2004;70(2):022318.

[40] Yang RG, Li N, Zhang J, Li J, Zhang TC. Enhanced entanglement of two optical modes in optomechanical systems via an optical parametric amplifier. J Phys At Mol Opt Phys. 2017;50(8):085502.

[41] Ahmed R, Qamar S. Optomechanical entanglement via nondegenerate parametric interactions. Phys Scr. 2017;92(10): 105101.

[42] Simon R. Peres-horodecki separability criterion for continuous variable systems. Phys Rev Lett. 2000 Mar;84(12):2726-9. 